\title{
Complicações e condutas fisioterapêuticas após cirurgia por câncer de mama: estudo retrospectivo
}

\author{
Complications and physical therapeutic treatment after breast cancer surgery: a \\ retrospective study
}

Simony Lira do Nascimento', Riza Rute de Oliveira', Mariana Maia Freire de Oliveira', Maria Teresa Pace do Amaral²

\begin{abstract}
RESUMOI Após cirurgia por câncer de mama, as mulheres estão sujeitas a desenvolver algumas complicações físicas. Os objetivos deste trabalho foram: investigar o desfecho dessas mulheres, que, durante o primeiro mês pós-operatório, foram submetidas a um programa de reabilitação e identificar ao longo de dois anos as complicações mais frequentes e as condutas fisioterapêuticas mais adotadas. Foi um estudo descritivo, retrospectivo, com dados de 707 prontuários de muIheres operadas por câncer de mama no Hospital da Mulher Professor Doutor José Aristodemo Pinotti da Universidade Estadual de Campinas, entre janeiro de 2006 e dezembro de 2007, atendidas pelo Setor de Fisioterapia. A análise foi feita por meio de médias, desvio padrão e frequências absolutas e relativas. Ao final do programa, 55\% das mulheres receberam alta, $17 \%$ necessitaram de atendimento adicional e $26 \%$ não aderiram a ele. As complicações mais frequentes foram: aderência pericicatricial (26\%), restrição da amplitude de movimento (24\%) e deiscência cicatricial (17\%). No primeiro ano após a cirurgia $(n=460)$, foram relatados dor $(28,5 \%)$, sensação de peso (21,5\%) e restrição da amplitude de movimento do ombro (16,7\%); já no segundo (n=168), houve dor (48,2\%), sensação de peso (42,8\%) e linfedema (23,2\%). Concluiu-se que, ao final do programa, a maioria das mulheres recebeu alta. Ao longo dos anos, houve redução da frequência de restrição da amplitude de movimento do ombro com aumento de linfedema. Cuidados com o braço, exercícios domiciliares e autodrenagem foram as condutas mais adotadas.
\end{abstract}

Descritores I fisioterapia; terapia por exercício; reabilitação; complicações pós-operatórias; câncer de mama.

\begin{abstract}
I After breast cancer surgery, women may develop some physical complications. Thus, the aims of this study were to investigate the outcome of these women, who participated in a rehabilitation program for one month, as well to identify along two years the most frequent complications and adopted physical therapy conducts. It was a descriptive and retrospective study with 707 medical records of women treated for breast cancer at the Women's Hospital Professor Doutor José Aristodemo Pinotti, Universidade Estadual de Campinas, between January 2006 and December 2007, admitted in the Division of Physical Therapy. Analysis was performed by means, standard deviation, absolute and relative frequencies. By the end of the program, 55\% of women were discharged, $17 \%$ required additional treatment, and $26 \%$ did not join it. The most frequent complications were: pericicatricial adherence (26\%), range of motion (ROM) restriction (24\%), and scar dehiscence (17\%). In the first year after surgery $(n=460)$, the main complaints were: pain (28.5\%), heaviness (21.5\%), and restriction of shoulder range of motion (16.7\%); in the second year ( $n=168$ ), they were pain (48.2\%), heaviness (42.8\%), and lymphedema (23.2\%). It was concluded that most women were discharged in the end of the program and, over the years, they presented reduction of shoulder range of motion frequency and lymphedema increase. Care of the arm, home exercises, and self-lymphatic drainage were the most adopted conducts.
\end{abstract}

Keywords I physical therapy; exercise therapy; rehabilitation; postoperative complications; breast cancer.

Estudo desenvolvido no Hospital da Mulher Professor Doutor José Aristodemo Pinotti (CAISM) da Universidade Estadual de Campinas (Unicamp) - Campinas (SP), Brasil.

'Fisioterapeutas; Doutorandas em Ciências da Saúde no Departamento de Tocoginecologia da Faculdade de Ciências Médicas da Unicamp - Campinas (SP), Brasil.

2Fisioterapeuta; Doutora em Ciências da Saúde no Departamento de Tocoginecologia da Faculdade de Ciências Médicas da Unicamp; Supervisora da Especialização de Fisioterapia Aplicada à Saúde da Mulher no Hospital da Mulher Professor Doutor José Aristodemo Pinotti da da Unicamp - Campinas (SP), Brasil. 


\section{INTRODUÇÃO}

As cirurgias por câncer de mama, bem como as terapias adjuvantes, podem resultar em algumas complicações físicas, dentre elas: infecção, necrose de pele, seroma, aderência e deiscência cicatriciais, limitação da amplitude de movimento (ADM) do ombro, cordão axilar, dor, alteração sensorial, lesão de nervos motor e/ou sensitivo, fraqueza muscular e linfedema ${ }^{1-7}$.

A fisioterapia é fundamental na reabilitação, prevenção e recuperação dos movimentos do membro superior no pós-operatório ${ }^{3,4,8-13}$, contribuindo para a melhora da conscientização corporal e oferecendo orientações necessárias para as atividades diárias ${ }^{3-5}$. Vários são os recursos fisioterapêuticos utilizados no pós-operatório de câncer de mama, entre eles a cinesioterapia, a terapia manual e o complexo descongestivo fisioterápico ${ }^{7,12,13}$.

Entretanto, a abordagem do câncer de mama pode ser complexa e dispendiosa, necessitando de medidas de qualidade para monitorar a eficácia deste acompanhamento e orientar as melhorias na saúde.

No Hospital da Mulher Professor Doutor José Aristodemo Pinotti (CAISM), na Universidade Estadual de Campinas (Unicamp), são atendidos aproximadamente 400 novos casos de câncer de mama por ano. A análise dos dados de um Serviço de Fisioterapia pertencente ao Centro, que é referência no tratamento do câncer de mama, permitiu avaliar o desfecho de um Programa de Reabilitação, durante o primeiro mês pós-operatório, e identificar por dois anos as complicações mais frequentes e condutas fisioterapêuticas mais adotadas. Tal conhecimento pode favorecer a implantação de melhores estratégias terapêuticas para as mulheres assistidas tanto no CAISM como em outros serviços.

\section{METODOLOGIA}

Estudo descritivo, retrospectivo, realizado por meio da consulta de 707 prontuários de mulheres submetidas à cirurgia por câncer de mama no CAISM, Unicamp, entre janeiro de 2006 e dezembro de 2007, encaminhadas para participar do Programa de Reabilitação de tal hospital. Foram excluídos os prontuários em que não foram encontradas as fichas de avaliação fisioterapêutica, ou quando a cirurgia realizada foi mastectomia simples (cirurgia em que não há necessidade de encaminhamento à fisioterapia).

Este estudo foi aprovado pelo Comitê de Ética em Pesquisa da Faculdade de Ciências Médicas da
Unicamp (1096/2009). Os dados de interesse foram coletados e anotados em uma ficha elaborada para este estudo e, posteriormente, digitalizados em planilha eletrônica (Excel). Foram realizadas análises descritivas, por meio de média, desvio padrão para variáveis contínuas e frequências absolutas e relativas para as variáveis nominais (n e \%).

\section{Programa de reabilitação}

No primeiro dia após a cirurgia, um fisioterapeuta orienta mulheres operadas a realizarem três exercícios para os membros superiores, englobando flexão, abdução e rotação de ombro. $\mathrm{Na}$ alta hospitalar, as mulheres recebem encaminhamento para o Programa de Reabilitação, que conta com equipe multidisciplinar (fisioterapia, enfermagem, psicologia e assistência social) além de cartilha informativa, contendo orientações sobre os cuidados com o membro superior homolateral à cirurgia ${ }^{14} \mathrm{e}$ instruções de exercícios para serem realizados em domicílio.

O Programa de Reabilitação ocorre durante o primeiro mês pós-operatório. Neste período são realizadas atividades em grupos (protocolados ou livres - constituídos de aproximadamente 19 exercícios para o membro superior, contendo alongamento, exercício ativo e relaxamento $)^{3,4,13,15}$, três vezes por semana, com duração aproximada de uma hora, por quatro semanas (12 sessões).

Ao final do programa, as mulheres são avaliadas por um fisioterapeuta e são reforçados os cuidados com o membro superior homolateral à cirurgia. Em tal momento, verifica-se a possibilidade de alta ou a necessidade de atendimento adicional.

\section{Reavaliações}

Reavaliações são agendadas conforme necessidade variando entre 3,6 ou 12 meses ou ainda durante a realização da radioterapia. Em algumas situações, quando a própria mulher reconhece a presença de complicação, ela procura reavaliação no Setor de Fisioterapia espontaneamente. Em todas as reavaliações os cuidados com o membro superior homolateral à cirurgia são reforçados, e a realização de exercícios diários é incentivada.

\section{Variáveis estudadas}

Avaliou-se o desfecho das mulheres após participação em tal programa, classificando-o em: alta, retorno ao Programa, atendimento individual (por meio de terapia manual), não adesão ao Programa (abandono ou 
não participação), encaminhamento para outro Serviço de Fisioterapia ou dispensada do Programa.

Também foram identificadas as queixas ou complicações apresentadas pelas mulheres ao final do Programa, no primeiro e segundo anos após a cirurgia. Dor (localizada em braço, axila ou região da mama), sensação de peso no membro superior, alteração sensorial (parestesias e/ou disestesias) e desconforto na realização de movimentos relatados como "me sinto amarrada", "sinto repuxão", "me sinto presa" foram considerados, de acordo com as queixas das mulheres. Foram avaliados também a presença de deiscência e/ou aderência pericicatricial, cordões axilares, $\mathrm{ADM}$ do ombro (flexão e abdução) e linfedema. As técnicas utilizadas foram a goniometria para verificação da ADM, a perimetria e a palpação para verificação do linfedema e inspeção e palpação para distúrbios cicatriciais, presença de cordões e alterações sensoriais.

As condutas fisioterapêuticas foram: cinesioterapia (exercício e alongamento ativos), terapia manual (massagem, pompagem, alongamento passivo, mobilização articular do ombro, entre outras) e complexo descongestivo fisioterapêutico (drenagem linfática manual, enfaixamento compressivo, exercícios ativos, cuidados com a pele e indicação de braçadeira elástica compressiva). Além disso, as mulheres receberam orientações quanto à realização de exercícios domiciliares, autodrenagem, autoenfaixamento e automassagem pericicatricial, além dos cuidados gerais com o membro superior homolateral à cirurgia.

\section{RESULTADOS}

A idade média das mulheres foi de 57 anos ( $\pm 13,7$ anos). Noventa e cinco por cento eram destras, $55 \%$ apresentaram sobrepeso (índice de massa corpórea - IMC>25 kg/m²), $18 \%$ eram diabéticas, $42 \%$ hipertensas e $10 \%$ apresentaram alguma disfunção musculoesquelética em ombro, previamente à cirurgia.

Os estadiamentos II e III foram apresentados por $63 \%$ das mulheres. A cirurgia mais frequente foi a mastectomia radical $(54,4 \%)$, seguida da quadrantectomia $(32,1 \%)$, sendo $52 \%$ destas realizadas na mama esquerda. Somente $15 \%$ das mulheres foram submetidas à reconstrução imediata, sendo que, em 50,5\%, utilizou-se o dermoexpansor (Tabela 1).

Das 707 mulheres incluídas neste estudo, 513 $(72,6 \%)$ aderiram ao Programa de Reabilitação. No primeiro ano após a cirurgia, 460 (65\%) operadas retornaram para avaliação. Destas, 62\% apresentaram alguma queixa ou complicação. Já no segundo ano, apenas 168 mulheres $(23,8 \%)$ retornaram ao Setor de Fisioterapia, das quais $83,3 \%$ apresentaram alguma queixa ou complicação (Figura 1).

Ao final do Programa de Reabilitação (um mês), 55\% das mulheres receberam alta, $17 \%$ continuaram por mais uma ou duas semanas no Programa ou receberam atendimento individualizado. Quinze por cento (15\%) não aderiram ao Programa, 5\% foram encaminhadas para realizar fisioterapia em outro Serviço e 6\% foram dispensadas do Programa por serem submetidas à mastectomia simples ou ampliação de margem cirúrgica (Tabela 2).

As complicações mais frequentes ao final do Programa foram aderência pericicatricial (26\%), restrição da ADM (24\%), deiscência da ferida cirúrgica (17\%) e cordões axilares (5\%), como pode ser observado na Tabela 2 .

A ADM média de flexão e abdução do ombro homolateral à cirurgia no pré-operatório foi de $173,7^{\circ}$ $\left( \pm 13,5^{\circ}\right)$ e $173,8^{\circ}\left( \pm 16,1^{\circ}\right)$, respectivamente. Após o Programa (um mês), estes valores foram $151,7^{\circ}\left( \pm 24,4^{\circ}\right)$ e $143,3^{\circ}\left( \pm 32^{\circ}\right)$. No primeiro ano após a cirurgia, a ADM média das mulheres foi de $163^{\circ}\left( \pm 19,1^{\circ}\right)$ para flexão e $162,3^{\circ}\left( \pm 24,8^{\circ}\right)$ para abdução; e no segundo ano, estes valores foram $155,1^{\circ}\left( \pm 22,6^{\circ}\right)$ e $152,2^{\circ}\left( \pm 27,8^{\circ}\right)$, respectivamente (Gráfico 1 ).

No primeiro ano, as complicações mais prevalentes foram dor (32,8\%), sensação de peso (21,5\%), restrição

Tabela 1. Principais características clínicas das mulheres submetidas a tratamento cirúrgico para câncer de mama

$\begin{array}{lcc}\text { Variáveis } & \text { n } & \% \\ \text { Lado operado } & & \\ \text { Direito } & 324 & 45,8 \\ \text { Esquerdo } & 365 & 51,6 \\ \text { Bilateral } & 18 & 2,5 \\ \text { Estadiamento clínico } & & \\ \text { In situ - I } & 228 & 32,2 \\ \text { II-III } & 445 & 62,9 \\ \text { IV } & 22 & 3,1 \\ \text { Sem informação } & 12 & 1,7 \\ \text { Tipo de cirurgia } & & \\ \text { Quadrante + biópsia do linfonodo sentinela } & 121 & 17,1 \\ \text { Quadrante + dissecção axilar } & 106 & 15 \\ \text { Mastectomia + biópsia do linfonodo sentinela } & 46 & 6,5 \\ \text { Mastectomia simples } & 36 & 5 \\ \text { Mastectomia simples + dissecção axilar } & 13 & 1,8 \\ \text { Mastectomia radical } & 385 & 54,4 \\ \text { Tipo de reconstrução imediata } & 103 & 14,5 \\ \text { Dermoexpansor } & 52 & 50,5 \\ \text { Retalho do músculo grande dorsal } & 26 & 25,2 \\ \text { Retalho do músculo retoabdominal } & 25 & 24,3 \\ \quad & & \\ & & \end{array}$




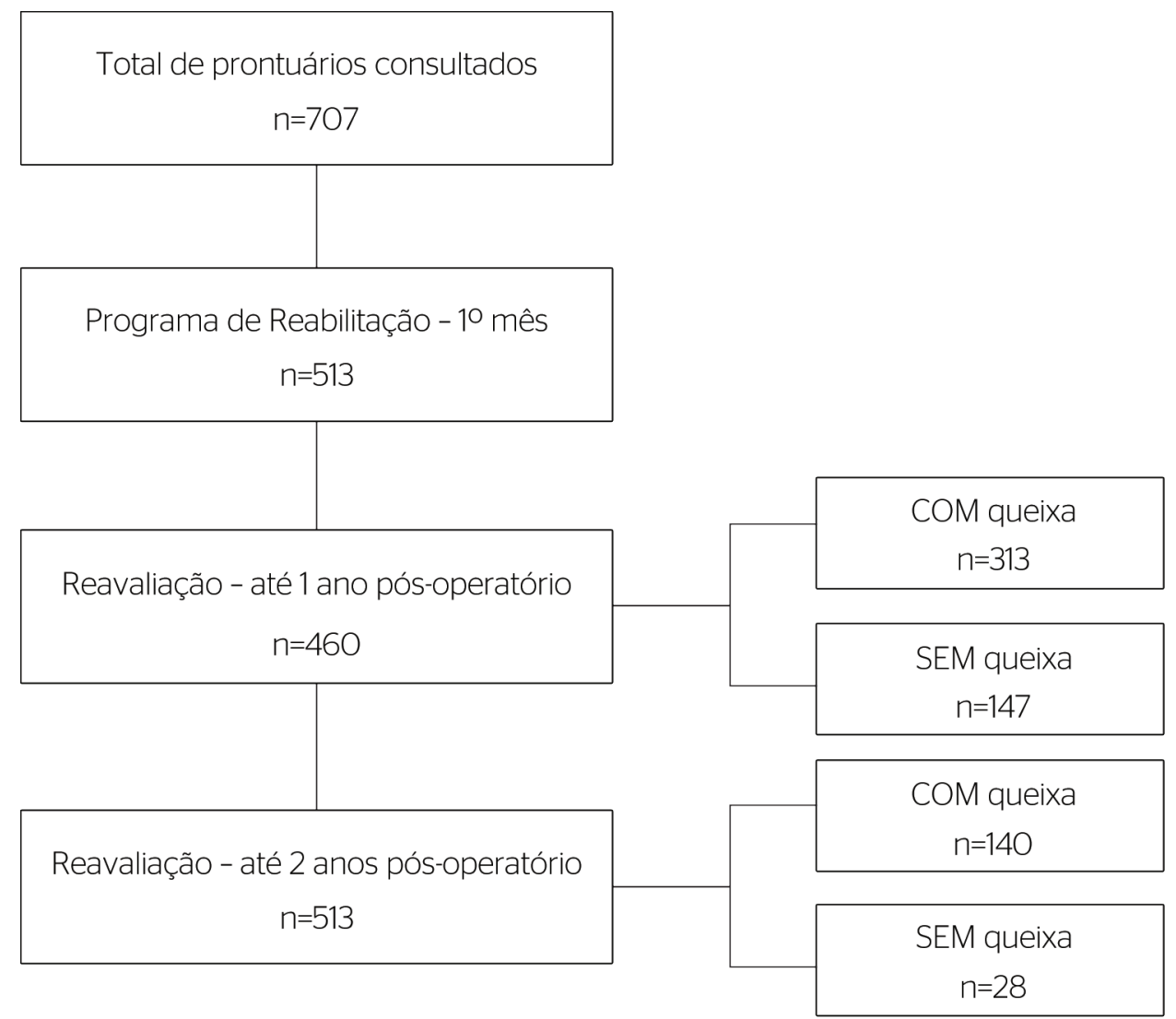

Figura 1. Fluxograma dos retornos das mulheres para avaliações fisioterapêuticas

Tabela 2. Desfecho, complicações e condutas

\begin{tabular}{|c|c|c|c|c|c|c|}
\hline \multirow{2}{*}{ Variáveis } & \multicolumn{2}{|c|}{ Final de 1 mês } & \multicolumn{2}{|c|}{ Até 1 ano } & \multicolumn{2}{|c|}{ Até 2 anos } \\
\hline & $\mathrm{n}$ & $\%$ & $\mathrm{n}$ & $\%$ & $\mathrm{n}$ & $\%$ \\
\hline \multicolumn{7}{|l|}{ Desfecho (ao final do programa de reabilitação) } \\
\hline Alta & 390 & 55,1 & - & - & - & - \\
\hline Retorno ao programa & 30 & 4,2 & - & - & - & - \\
\hline Atendimento individual & 81 & 11,4 & - & - & - & - \\
\hline Retorno ao programa e atendimento individual & 12 & 1,7 & - & - & - & - \\
\hline Não adesão ao programa & 104 & 14,7 & - & - & - & - \\
\hline Encaminhamento a outro serviço & 33 & 4,66 & - & - & - & - \\
\hline Dispensada do programa & 44 & 6,22 & - & - & - & - \\
\hline Sem informação & 13 & 1,83 & - & - & - & - \\
\hline \multicolumn{7}{|l|}{ Complicações (ao final do programa de reabilitação) } \\
\hline Restrição de ADM & 172 & 24,3 & - & - & - & - \\
\hline Cordão axilar & 37 & 5,2 & - & - & - & - \\
\hline Aderência cicatricial & 181 & 25,6 & - & - & - & - \\
\hline Deiscência cicatricial & 118 & 16,7 & - & - & - & - \\
\hline \multicolumn{7}{|l|}{ Condutas } \\
\hline Automassagem pericicatricial & - & - & 50 & 10,9 & 15 & 8,9 \\
\hline Atendimento individual & - & - & 79 & 17,2 & 13 & 7,7 \\
\hline Autodrenagem & - & - & 99 & 21,5 & 80 & 47,6 \\
\hline Complexo descongestivo fisioterapêutico & - & - & 6 & 1,3 & 16 & 9,5 \\
\hline Outras condutas* & - & - & 92 & 20 & 33 & 19,6 \\
\hline
\end{tabular}

ADM: amplitude de movimento; *crioterapia, cuidados com a pele (hidratação), reeducação postural, dessensibilização, orientação sobre a braçadeira e estimulação nervosa elétrica transcutânea (transcutaneous electrical nerve stimulation - TENS) 
da $\operatorname{ADM}(16,7 \%)$, alteração sensorial (14,8\%), aderência pericicatricial $(10,8 \%)$ e linfedema $(6,1 \%)$. Já no segundo ano, foram dor $(48,2 \%)$, sensação de peso no braço (42,8\%), linfedema (23,2\%), restrição da $\operatorname{ADM}(17,8 \%)$ e alteração sensorial (11,9\%), como observado no Gráfico 2.

Outras complicações comumente relatadas pelas mulheres ao longo dos dois anos foram: pele ressecada, dor por metástases, efeitos colaterais da quimioterapia, radiodermite, complicações da reconstrução mamária (necrose ou infecção do sítio cirúrgico), bursites e tendinites no ombro.

Nas avaliações durante o primeiro ano, verificou-se que $73 \%$ das mulheres relataram realizar os exercícios domiciliares frequentemente e $75 \%$ seguiam as orientações sobre

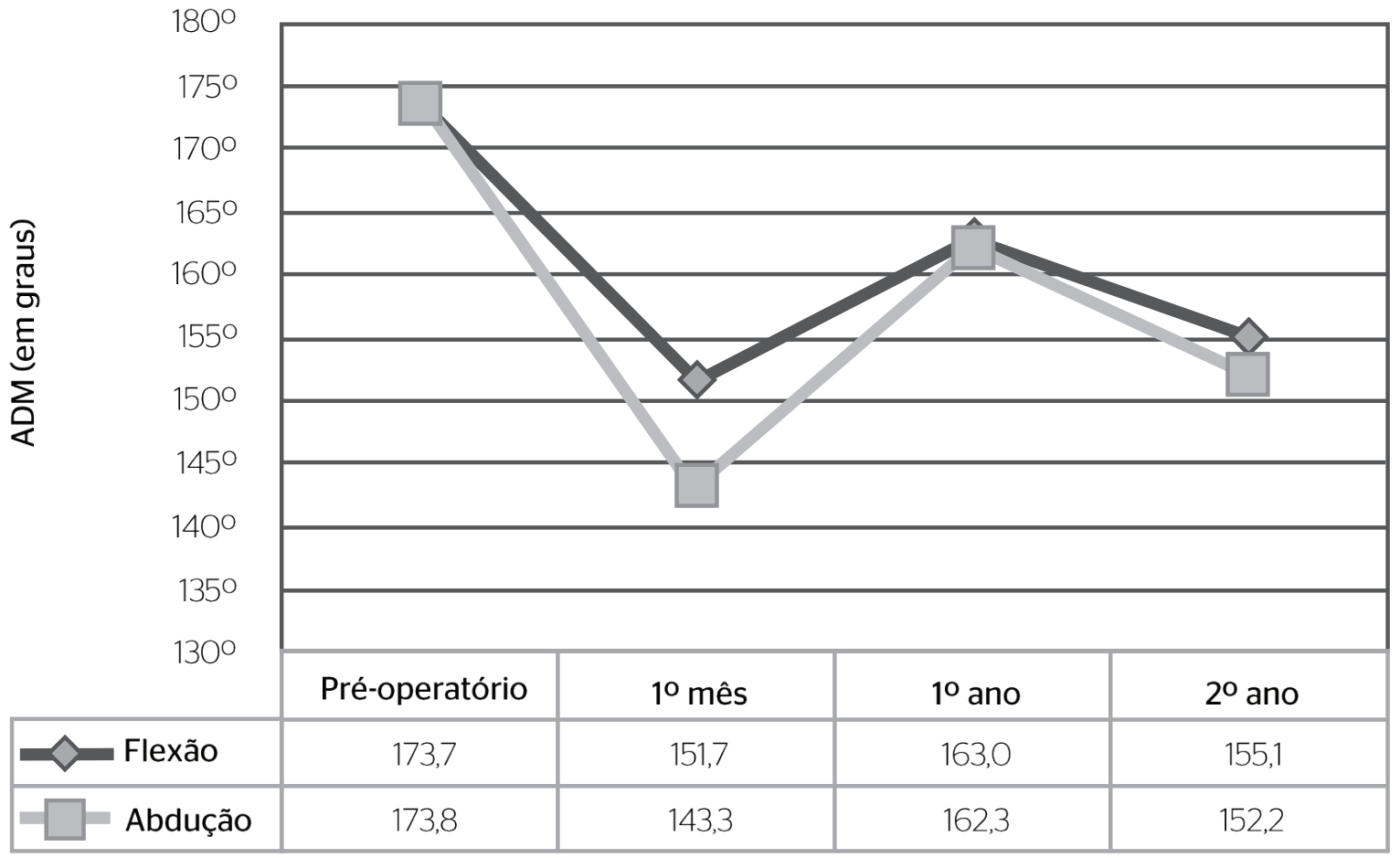

ADM: amplitude de movimento

Gráfico 1. Evolução das amplitudes de flexão e abdução do ombro, em graus, durante dois anos, sendo n no pré-operatório de 707; em um mês, 513; primeiro ano, 460 e segundo ano, 168

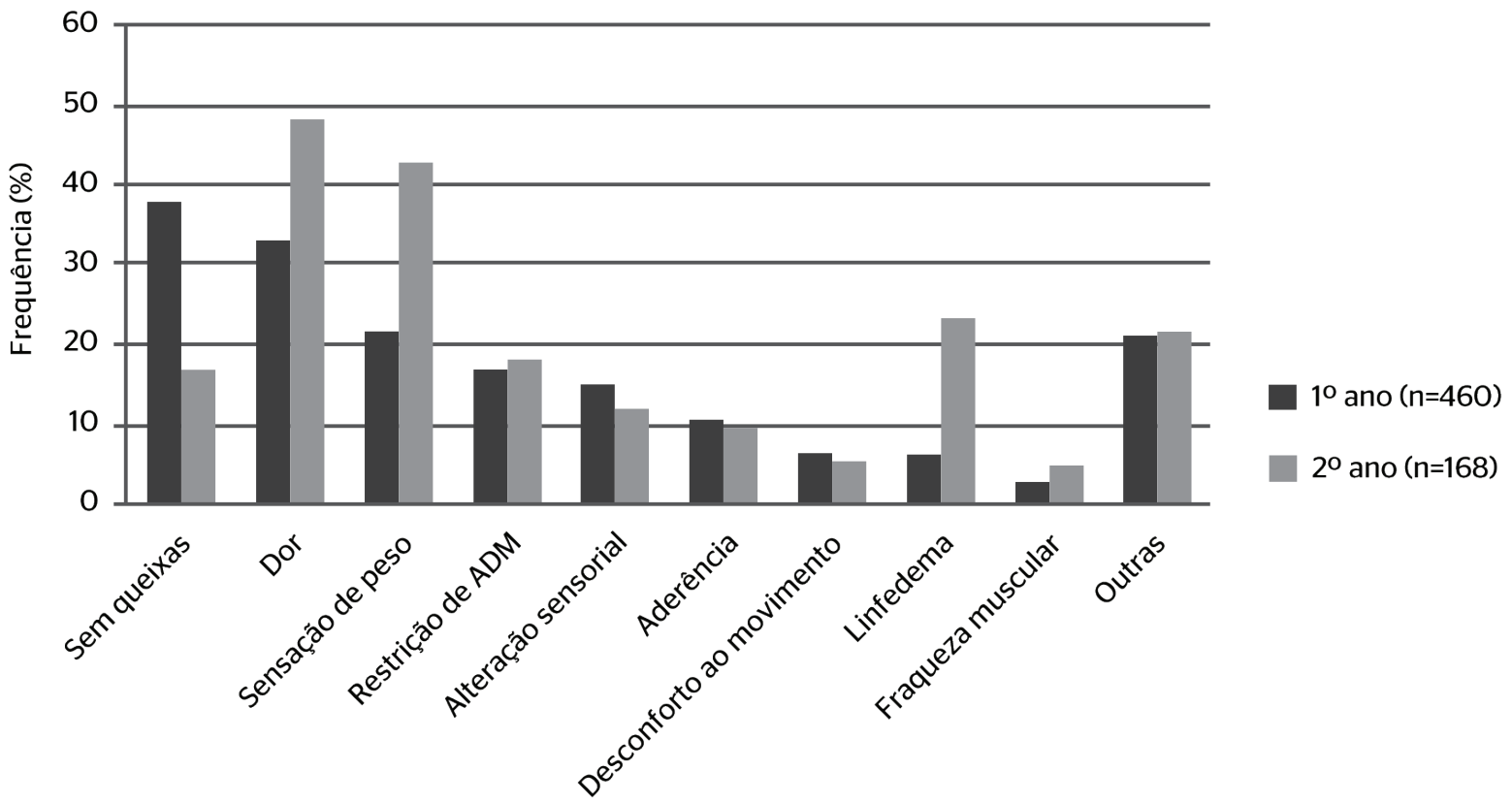


os cuidados com o membro homolateral à cirurgia. Em relação às condutas fisioterapêuticas adotadas no primeiro ano, todas as mulheres receberam orientações para dar continuidade aos exercícios e cuidados com o membro superior. A autodrenagem foi orientada a $21,5 \%$ das mulheres e massagem pericicatricial a $10,9 \%$ delas. Para $17,2 \%$ das mulheres, empregou-se a terapia manual e, para $1,3 \%$, o complexo descongestivo fisioterapêutico (Tabela 2).

No segundo ano após a cirurgia, as mulheres receberam recomendações para continuarem com os exercícios e cuidados com o membro superior. Ainda neste período, a autodrenagem foi orientada a $47,6 \%$ das mulheres e a massagem pericicatricial a $8,9 \%$ delas. Foram tratadas por meio da terapia manual $7,7 \%$ das mulheres e $9,5 \%$ se submeteram ao complexo descongestivo fisioterapêutico (Tabela 2).

\section{DISCUSSÃO}

Considerando o Programa de Reabilitação, assim como na literatura ${ }^{3,4,10,11,15-18}$, observou-se a eficácia da fisioterapia ao ser constatado no presente Serviço que a maioria das mulheres apresentou ADM funcional e ausência de complicações, recebendo, portanto, alta fisioterapêutica ao final do Programa.

Também foi notado que aproximadamente $15 \%$ das mulheres não aderiram ao Programa, impossibilitando o conhecimento da real condição fisicofuncional apresentada por elas durante o período estudado. Como este hospital é um local de referência no tratamento de câncer de mama, muitas mulheres de cidades e estados vizinhos buscam-no para se tratarem. Possivelmente, estas mulheres não participaram do Programa por residirem distante do hospital, em alguns casos, optaram por buscar auxílio em organizações não governamentais (ONGs) existentes nos municípios próximos de suas casas. De acordo com Gutiérrez et al., outros motivos, como medo de comprometer o processo cicatricial, de sentir dor ao realizar os exercícios ou ainda a falta de ânimo e/ou mal-estar, também podem influenciar na não adesão ${ }^{19}$.

Apesar dos diversos fatores que podem afastar a mulher do atendimento periódico dentro do CAISM, faz parte do protocolo deste Programa, no momento da alta hospitalar, a orientação quanto aos cuidados gerais com o membro operado, assim como a orientação de retorno ao Serviço de Fisioterapia sempre que necessário, ainda que seja em data coincidente com as consultas médicas.

Dentre as mulheres que apresentaram algum prejuízo funcional ao final do Programa, destacam-se os seguintes fatores causais:
- aderência - consequente ao comprometimento do deslizamento miofascial, especialmente na cicatriz cirúrgica, nas áreas irradiadas e nas regiões escapular e lateral do tórax ${ }^{20}$;

- deiscência cicatricial - normalmente tem incidência entre 10 a $22 \%{ }^{15}$;

- cordões axilares - acarreta dor e limitação da ADM, com prevalência variável de 6 a 72\% $\%^{6,21,22}$.

Além destes fatores, ainda foram encontradas complicações como dor, disfunções musculoesqueléticas no ombro homolateral à cirurgia, ansiedade e medo.

No primeiro ano após a cirurgia, a ADM do ombro melhorou em relação aos valores verificados ao final do Programa, possivelmente pela eliminação dos efeitos agudos do tratamento cirúrgico e/ou radioterápico. Esse achado é compatível com a literatura, que evidencia melhora da ADM após um período de três meses a um ano ${ }^{10,16,17,23}$. A dor na região da mama ou no membro superior foi a queixa mais frequente no período estudado e, segundo a literatura, sua frequência pode variar de 12 a $39 \%$ no primeiro ano após a cirurgia ${ }^{11,24}$.

Após dois anos da cirurgia, verificou-se diminuição da ADM de ombro em 17,8\% das mulheres que retornaram ao Serviço. Tal fato não significa que essa amplitude de todas as mulheres operadas se reduz com o tempo, mas pode estar relacionada a menor amostra avaliada neste período, que corresponde às mulheres que buscaram o Setor de Fisioterapia para avaliação por apresentarem alguma queixa e/ou complicação física, dentre elas a limitação do movimento. Acredita-se que as demais não retornaram por apresentarem boa condição fisicofuncional.

Ainda no segundo ano de cirurgia, além da restrição da ADM, verificou-se aumento da frequência de linfedema, ambos são impactantes na morbidade física por prejudicarem a mobilidade do membro superior e interferirem nas atividades de vida diária e psicológica, por alterar a autoimagem ${ }^{25}$. Sagen et al. relataram frequência de linfedema de $5 \%$ após três meses de cirurgia, com aumento para $12 \%$ em dois anos, e observaram que mulheres com IMC pré-operatório maior que $25 \mathrm{~kg} / \mathrm{m}^{2}$ têm três vezes mais chance de desenvolver linfedema ${ }^{26}$.

A maioria das mulheres deste estudo exercia atividade laboral que exigia grande esforço físico (diarista, doméstica ou do lar), inclusive do membro operado, e muitas vezes não encontravam outras pessoas que pudessem substituí-las nesses cargos. Desta forma, os cuidados necessários para evitar complicações eram reduzidos, podendo contribuir no surgimento de algumas complicações, como dor e linfedema. 
Em geral, as complicações citadas podem levar a um prejuízo importante na funcionalidade do membro operado, comprometendo a qualidade de vida e o tratamento complementar. A impossibilidade ou dificuldade de posicionamento adequado do ombro também podem retardar o início da radioterapia. Portanto, é necessário estar atento ao diagnóstico para uma possível intervenção nestas complicações.

No que diz respeito ao atendimento prestado no Serviço de Fisioterapia do CAISM, preconiza-se pela continuidade do acompanhamento fisioterapêutico individualizado, utilizando-se a terapia manual como principal recurso em casos de persistência de dor e limitação de ADM ao final do Programa. A terapia manual é direcionada para restaurar os movimentos da superfície articular, com o objetivo de tratar a dor por meio da ativação de estruturas neurais, além de favorecer o alongamento do tecido conjuntivo e, consequentemente, a melhora do movimento fisiológico ${ }^{27}$.

O segundo recurso manual mais utilizado neste Centro é a massagem pericicatricial, que, associada à mobilização articular, também pode estimular a propriocepção, favorecendo o movimento ${ }^{28}$ e promovendo melhor qualidade da cicatriz e alívio na sensibilidade ${ }^{29}$. Em relação ao surgimento de complicações tardias como sensação de peso e linfedema, preconiza-se a indicação de autodrenagem e do complexo descongestivo fisioterápico. Dessensibilização, crioterapia, hidratação da pele, indicação de braçadeira elástica compressiva e reeducação postural também são condutas adotadas pelo Serviço de Fisioterapia, de acordo com a necessidade de cada mulher.

Alguns aspectos limitaram os resultados deste estudo, como a não adesão de todas as mulheres ao Programa de Reabilitação, o número de retornos variados, as avaliações realizadas por diferentes fisioterapeutas e a coleta feita retrospectivamente. Apesar disso, a grande demanda de mulheres operadas por câncer de mama, existente no CAISM, associada à descrição de um Serviço de Fisioterapia de referência servem como parâmetros para outros serviços e fisioterapeutas. Ainda, pode contribuir para o planejamento de melhorias na atenção às mulheres submetidas à cirurgia por câncer de mama e também na inovação de estratégias terapêuticas.

\section{CONCLUSÕES}

Ao final do Programa a maioria das mulheres recebeu alta, demonstrando que esta estratégia de reabilitação do hospital favoreceu a recuperação fisicofuncional para a maioria das mulheres. Ao longo dos anos, houve redução na frequência de restrição da ADM, com aumento na frequência de linfedema. Cuidados com o membro operado, exercícios domiciliares e autodrenagem foram as condutas mais adotadas. Portanto, verificou-se que a inserção de mulheres em um Programa de Reabilitação contribui de forma eficaz no processo de reabilitação, na prevenção e no tratamento de complicações após cirurgia por câncer de mama.

\section{REFERÊNCIAS}

1. Wallace MS, Wallace AM, Lee J, Dobke MK. Pain after breast surgery: a survey of 282 women. Pain. 1996;66(2-3):195-205.

2. Warmuth MA, Bowen G, Prosnitz LR, Chu L, Broadwater G, Peterson B, et al. Complications of axillary lymph node dissection for carcinoma of the breast. Cancer. 1998;83(7):1362-8.

3. Silva MP, Derchain SF, Rezende L, Cabello C, Martinez EZ. Movimento do ombro após cirurgia por carcinoma invasor da mama: estudo randomizado prospectivo controlado de exercícios livres versus limitados a 90 no pós-operatório. Rev Bras Ginecol Obst. 2004;26(2):125-30.

4. Amaral MT, Teixeira LC, Derchain SF, Nogueira MD, Silva MP, Gonçalvez AV. Orientação domiciliar: proposta de reabilitação física para mulheres submetidas à cirurgia por câncer de mama. Rev Cienc Med. 2005;14(3):405-13.

5. Barranger E, Dubernard G, Fleurence J, Antoine M, Darai E, UzanS. Subjective morbidity and quality of life after sentinel node biopsy and axillary lymph node dissection for breast cancer. J Surg Oncol. 2005;92(1):17-22.

6. Bergmann A, Mattos IE, Koifman RJ, Ribeiro MJ, Nogueira EA, Ribeiro EP, et al. Axillary web syndrome after lumph node dissection: results of 1004 breast cancer patients. Lymphology. 2007;40(suppl):198-203.

7. Cheville AL, Tchou J. Barriers to rehabilitation following surgery for primary breast cancer. J Surg Oncol. 2007;95(5):409-18.

8. Hayes S, Battistutta D, Newman B. Objective and subjective upper body function six months following diagnosis of breast cancer. Breast Cancer Res Treat. 2005;94(1):1-10.

9. Rietmam JS, Dijkstra PU, Geertzen JH, Baas P, De Vries J, Groothoff JW, et al. Short-term morbidity of the upper limb after sintinel lymph node biopsy or axillary lymph node dissection for stage I or II breast carcinoma. Cancer. 2003;98(4):690-6.

10. Box RC, Reu-Hirche HM, Bullock-Saxton JE, Furnival CM. Shoulder movement after breast cancer surgery: results of a randomized controlled study of postoperative physiotherapy. Breast Cancer Res and Treat. 2002;75(1):35-50.

11. Karki A, Simonen R, Malkia E, Selfe J. Impairments, activity limitations and participation restrictions 6 and 12 months after breast cancer operation. J Rehabil Med. 2005;37(3):180-8.

12. McAnaw MB, Harris KW. The role of physical therapy in rehabilitation of patients with mastectomy and breast reconstruction. Breast Disease. 2002;16:163-74.

13. Rezende LF, Franco RL, Gurgel MS. Fisioterapia aplicada à fase pósoperatória de câncer de mama: O que considerar? Rev Cienc Med. 2005;14(3):295-302. 
14. Bergmann A, Ribeiro MJ, Pedrosa E, Nogueira EA, Oliveira AC. Fisioterapia em mastologia oncológica: rotinas do Hospital do Câncer III/INCA. Rev Bras Cancerol. 2006;52(1):97-109.

15. Rezende LF, Beletti PO, Franco RL, Moares SS, Gurgel MS. Exercícios livres versus direcionados nas complicações pós-oparatórias de câncer de mama. Rev Assoc Med Bras. 2006;52(1):37-42.

16. Lauridsen MC, Christiansen P, Hessov R. The effect of physiotherapy on shoulder function in patients surgically treated for breast cancer: a randomized study. Acta Oncol. 2005;44(5):449-57.

17. Beurskens $\mathrm{CH}$, van Uden CJ, Strobbe LJ, Oostendorp RA, Wobbes $T$. The efficacy of physiotherapy upon shoulder function following axillary dissection in breast cancer, a randomized controlled study. BMC Cancer. 2007;7:166

18. McNeely ML, Campbell K, Ospina M, Rowe BH, Dabbs K, Klassen TP, et al. Exercise interventions for upper-limb dysfunction due to breast cancer treatment. Cochrane Database Syst Rev. 2010;16(6):CD005211.

19. Guitérrez MG, Bravo MM, Chanes C, de Vivo MC, Souza GO. Adesão de mulheres mastectomizadas ao início precoce de um programa de reabilitação. Acta Paul Enferm. 2007:20(3):249-54.

20. Fourie WJ. Considering wider myofascial involvement as a possible contributor to upper extremity dysfunction following treatment for primary breast cancer. J Bodyw Mov Ther. 2008;12(4):349-55.

21. Moskovitiz AH, Anderson BO, Yeung RS, Byrd DR, Lawton TJ, Moe RE. Axillary web syndrome after axillary dissection. Am J Surg. 2001:181(5):434-9.
22. Leidenius M, Leppanen E, Krogerus L, von Smitten K. Motion restriction and axillary web syndrome after sentinel node biopsy and axillary clearance in breast cancer. Am J Surg. 2003:185(2):127-30.

23. Oliveira MM, Gurgel MSC, Miranda MS, Okubo MA, Feijó LF, Souza GC. Eficácia dos exercícios para ombro nas complicações loco-regionais em mulheres submetidas a radioterapia para câncer de mama: ensaio clínico. Rev Bras Fisioter. 2008;13(2):136-43.

24. Thomas-MacLean RL, Hack TH, Kwan W, Towers A, Miedema B, Tilley A. Arm morbidity and disability after breast cancer: new directions for care. Oncol Nurs Forum. 2008;35(1):65-71.

25. Freitas Jr R, Ribeiro LF, Taia L, Kajita D, Fernandes MV, Queiroz GS. Linfedema em pacientes submetidas à mastectomia radical modificada. Rev Bras Ginecol Obstet. 2001;23(4):205-8.

26. Sagen A, Karensen R, Risberg MA. Physical activity for the affected limb and arm lymphedema after breast cancer surgery. A prospective, randomized controlled trial with two years follow-up. Acta Oncol. 2009:48(8):1102-10

27. Threlkeld AJ. The effects of manual therapy on connective tissue. Phys Ther. 1992;72(12):893-902

28. Senbursa G, Baltaci G, Atay A. Comparison of conservative treatment with and without manual physical therapy for patients with shoulder impingement syndrome: a prospective, randomized clinical Trial. Knee Surg Sports Traumatol Arthrosc. 2007;15(7):915-21.

29. Roque C. Massage applied to scars. Wound Repair Regen. 2002:10(2):126-8 